\title{
Incidence features of second primary malignancy among gastric cancer survivors, 1992-2012
}

\author{
Shuai Li ${ }^{1 \#}$, Yiyang Luo ${ }^{2 \#}$, Qi Hou ${ }^{1}$, Huaqing Chu ${ }^{1}$, Hui Zheng ${ }^{1}$ \\ ${ }^{1}$ Department of Anesthesiology, National Cancer Center/Cancer Hospital, Chinese Academy of Medical Sciences and Peking Union Medical \\ College, Beijing, China; ${ }^{2}$ Department of Hysteroscopic Center, Fuxing Hospital, Capital Medical University, Beijing, China \\ Contributions: (I) Conception and design: S Li, H Zheng; (II) Administrative support: H Zheng; (III) Provision of study materials or patients: S Li; (IV) \\ Collection and assembly of data: Y Luo, Q Hou; (V) Data analysis and interpretation: S Li, H Zheng, H Chu; (VI) Manuscript writing: All authors; \\ (VII) Final approval of manuscript: All authors. \\ "These authors contributed equally to this work. \\ Correspondence to: Prof. Hui Zheng. Department of Anesthesiology, National Cancer Center/Cancer Hospital, Chinese Academy of Medical Sciences \\ and Peking Union Medical College, Beijing, China. Email: 13796657128@126.com.
}

Background: With the expanding population of cancer survivors, screening for second primary malignancy (SPM) is one issue concerning survivorship care. This study aimed to evaluate the potential risk of developing SPMs and determine the features of SPMs among patients after the diagnosis of gastric cancer (GC).

Methods: We identified 33,705 GC patients from the Surveillance, Epidemiology, and End Results Program (SEER) database. The standardized incidence ratios (SIRs) were calculated to estimate the risk of SPMs. The SIRs were stratified by age, race, stage, and latency period since GC diagnosis.

Results: A total of 2,018 among 33,705 GC patients developed SPMs. Compared with the general population, these GC survivors had higher risks of developing second malignancies of the esophagus, stomach, small intestine, colon, pancreas, and thyroid. Conversely, these GC survivors had lower chances of developing prostate and female breast cancer.

Conclusions: In stratified analyses, we identified the features associated with a higher risk of developing SPMs, including age between 20 and 39 years, latency between 60 and 119 months, localized stage, and American Indian/Alaska Native ethnicity. Hence, GC survivors are at a higher risk of developing SPMs than the general population. Careful attention and continuous surveillance should be used when treating these patients.

Keywords: Incidence; second primary malignancy (SPM); gastric cancer survivors (GC survivors); 1992-2012

Submitted Feb 20, 2020. Accepted for publication Sep 27, 2020.

doi: $10.21037 /$ tcr-20-1105

View this article at: http://dx.doi.org/10.21037/tcr-20-1105

\section{Introduction}

According to the World Cancer Report 2014, gastric cancer (GC) has the fifth leading incidence of cancers and is the third leading cause of death from cancer. Approximately 951,600 new GC cases were diagnosed and 723,100 GCrelated deaths occurred in 2012 (1). Hence, GC is currently a significant public health issue in the world.

Although the GC incidence and mortality rates are generally declining in many countries, GC survivors still account for a large proportion of all cancer patients $(2,3)$. With the large population of GC survivors, one concerning issue is their long-term survival status, especially the risk of developing second primary malignancies (SPMs) $(4,5)$. Previous studies have reported that the risk of developing an SPM is increased in several types of cancer (6-8). Accumulating evidence has suggested that healthy aging, intensive treatment of the primary tumor, and genetic and 
environmental factors are significant risk factors for an elevated incidence of SPM $(6,9)$. Therefore, it is essential to determine the risk of SPM among GC survivors and then provide them with continuous cancer surveillance.

In this retrospective study, we used data from the Surveillance Epidemiology and End Results (SEER) cancer registry and determined the risk of SPM after a diagnosis of GC in the United States between 1992 and 2012 (10). We aimed to identify the epidemiological and clinical features of first primary GC related to the occurrence of SPM. We present this study in accordance with the STROBE reporting checklist (available at http://dx.doi.org/10.21037/ tcr-20-1105).

\section{Methods}

\section{Date source}

We collected patient information from the SEER 13 database, which includes processed publicly available data collected between 1992 and 2012 from 13 registries (the New Mexico, San Francisco-Oakland, Atlanta, San JoseMonterey, rural Georgia, Connecticut, Detroit, Hawaii, Iowa, Utah, Los Angeles, Seattle-Puget Sound, and Alaska Native Tumor Registries). The SEER database represents approximately $28 \%$ of the US population. The SEER program provides data on patient demographics, cancer incidence, tumor characteristics, limited therapeutic information, and survival data. To distinguish SPM from recurrence and metastases of the primary GC, the SEER system strictly follows the coding rules of histology and topography classification of the International Classification of Diseases for Oncology third edition (ICD-O-3).

The study design was approved by the Ethics Committee of National Cancer Center/Cancer Hospital, Chinese Academy of Medical Sciences and Peking Union Medical College. The SEER database is open access. Therefore, the authors can obtain cancer case and population data from the SEER website. Patients' records were anonymized and deidentified prior to analysis.

\section{Study population}

All patients had histologically confirmed GC, which included only one primary GC tumor as well as the primary GC in patients with multiple primary malignancies. We collected cases of invasive GC diagnosed in patients at an age of more than 20 years to ensure that young patients could be captured in this study. We excluded cases of primary GC with the following attributes: (I) unknown diagnosis age, (II) in situ tumors, and (III) report of primary GC on only the death or autopsy certificate. In addition, SPMs diagnosed during the 6-month period after the primary diagnosis were also excluded to minimize misclassification of undetected synchronous cancers and metastases.

\section{Statistical analyses}

Multiple primary standardized incidence ratios (SIRs) were calculated as one measure to estimate the relative risk of SPM. The SIR was defined as the ratio of the observed incidence of SPM among GC patients to the expected incidence in the US general population within the SEER database. Statistical significance of SIRs was defined at a $\mathrm{P}$ value $<0.05$ (two-sided). In addition, 95\% confidence intervals (CIs) were calculated as exact Poisson confidence limits for the ratio of observed events to expected events. Analyses in this study were stratified by age at primary GC diagnosis (20-39, 40-59, or $\geq 60$ years), race (white, black, American Indian/Alaska Native, Asian or Pacific Islander), stage (localized, regional, or distant), and latency period since primary GC diagnosis $(6-11,12-59,60-119$, or $\geq 120$ months). The definitions of tumor stage in the SEER database are as follows: localized stage, an invasive neoplasm confined entirely to the organ of origin; regional stage, a neoplasm that has extended beyond the limits of the organ of origin directly into surrounding organs and tissue or into regional lymph nodes; and distant stage, a neoplasm that has spread to parts of the body remote from the primary tumor, such as distant organs, tissues or distant lymph nodes. All analyses were conducted using SEER*Stat software version 8.2.1. The study was conducted in accordance with the Declaration of Helsinki (as revised in 2013).

\section{Results}

\section{Study population characteristics}

In this study, we identified 33,705 eligible individuals who were diagnosed with primary GC. The majority of patients diagnosed with primary GC were male $(60.7 \%)$, aged $>60$ years $(68.3 \%)$, and white $(66.6 \%)$. Among all patients, 2,018 patients $(6.0 \%)$ subsequently developed SPMs. For patients who developed SPMs, $86.6 \%$ were aged $>60$ years, and $40.7 \%$ of patients had localized-stage tumors; 
Table 1 Variables of GC patients who developed SPMs

\begin{tabular}{|c|c|c|}
\hline Variable & $\begin{array}{l}\text { Patients with first primary GC } \\
\qquad(n=33,705), n(\%)\end{array}$ & $\begin{array}{l}\text { GC patients who developed SPMs } \\
\qquad(n=2,018), n(\%)\end{array}$ \\
\hline \multicolumn{3}{|l|}{ Sex } \\
\hline Female & $13,235(39.3)$ & $689(34.2)$ \\
\hline $40-59$ & $9,218(27.3)$ & 260 (12.9) \\
\hline$\geq 60$ & 23,007 (68.3) & $1,747(86.6)$ \\
\hline \multicolumn{3}{|l|}{ Race } \\
\hline Asian or Pacific Islander & $7,108(21.1)$ & $385(19.1)$ \\
\hline \multicolumn{3}{|l|}{ Calendar year } \\
\hline 1992-2001 & $15,749(46.7)$ & $492(24.4)$ \\
\hline 2002-2012 & $17,956(53.3)$ & $1,526(75.6)$ \\
\hline \multicolumn{3}{|l|}{ Stage at GC diagnosis } \\
\hline Localized & $7,693(22.8)$ & $821(40.6)$ \\
\hline Regional & $9,168(27.3)$ & $313(15.5)$ \\
\hline $60-119$ & - & $584(28.9)$ \\
\hline$\geq 120$ & - & $280(13.9)$ \\
\hline
\end{tabular}

in addition, $15.5 \%$ of patients had regional-stage tumors, and $16.6 \%$ of patients had distant-stage tumors (16.6\%). A more detailed overview of patient characteristics is shown in Table 1 .

\section{SIRs for SPMs}

Compared with the general population, GC patients had higher overall risk for SPM (SIR 1.11, 95\% CI: 1.06-1.16). Patients with GC had significantly increased risks for malignancies of the stomach (SIR 4.51, 95\% CI: 3.93-5.16), small intestine (SIR 3.06, 95\% CI: 1.96-4.55), esophagus
(SIR 2.41, 95\% CI: 1.79-3.17), colon (SIR 1.18, 95\% CI: 1.01-1.37), pancreas (SIR 1.60, 95\% CI: 1.29-1.96) and thyroid (SIR 2.00, 95\% CI: 1.37-2.80) than the general population. However, the risk of SPMs involving the female breast (SIR 0.79, 95\% CI: 0.66-0.95) and prostate (SIR $0.79,95 \% \mathrm{CI}: 0.70-0.89)$ were decreased in the primary GC population. The detailed data are shown in Table 2 .

\section{Age at first GC diagnosis}

Age is deemed one crucial risk factor for GC patients, so 
Table 2 Risk of SPMs in patients with GC

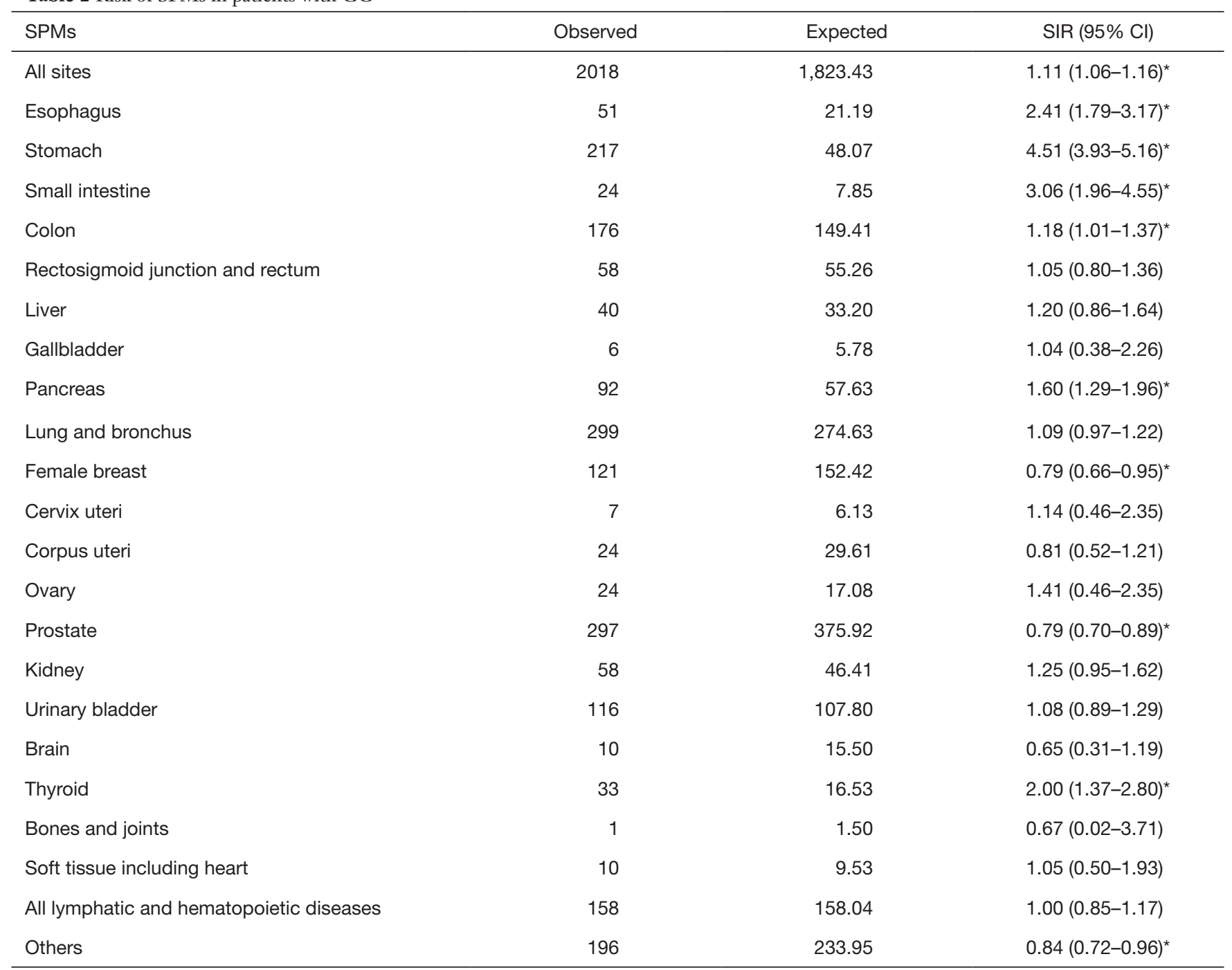

*, $\mathrm{P}<0.05$.

we compared the SIRs in three different age subgroups, including 20-39, 40-59, and $\geq 60$ years, to determine whether the risk of SPMs varied with age. The results showed that GC patients aged 20-39 and 40-59 years had predominantly high risks of developing SPMs in all sites (Figure 1A). Patients aged 20-39 years had a significantly higher risk of developing SPMs of the stomach (SIR 50.19) and colon (SIR 10.41) and patients aged 40-59 had a higher risk of developing malignancies of the esophagus, stomach, colon, pancreas, prostate, lung, and bronchus than patients in the other two age groups. Although the risk was not observed in all sites, patients aged $>60$ years showed an increased risk for SPMs involving the esophagus, stomach, small intestine, pancreas, and thyroid (Table 3).

\section{Race}

There were significant changes in SIRs between different races for SPMs at all sites (Figure $1 B$ ). The highest risk for SPMs was in American Indian/Alaska Native patients, followed by that in black and white patients. The risk of developing SPM of the stomach was higher in these four races than that in other races. In addition, the risk of esophageal SPM was elevated in black patients, and the risk of small intestinal SPM was elevated in American Indian/ Alaska Native patients. For white GC patients, there was 

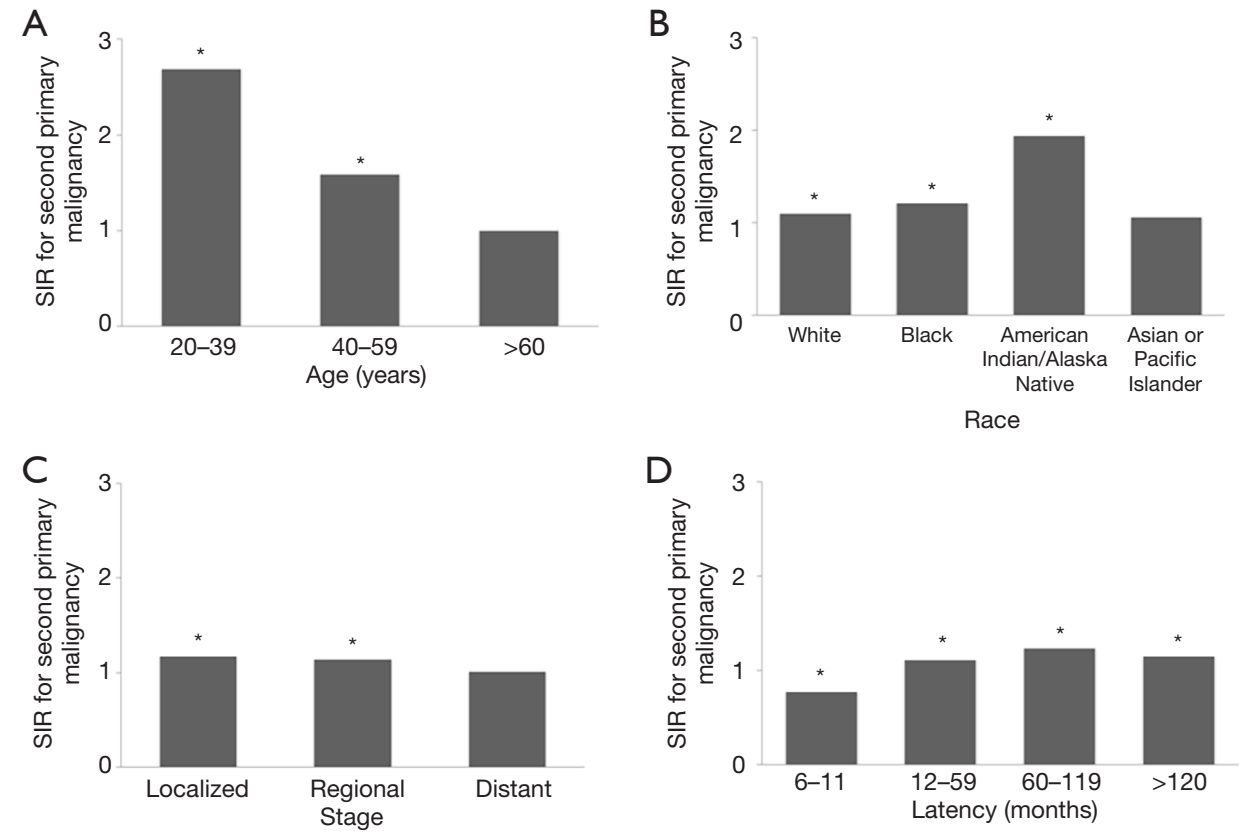

Figure 1 SIRs of SPMs in GC patients. (A) SIRs of SPMs in GC patients by age; (B) SIRs of SPMs in GC patients by race; (C) SIRs of SPMs in GC patients by stage; (D) SIRs of SPMs in GC patients by latency. *, the observed incidence of SPMs among SC is significantly lower or higher than the expected incidence in US general population, with $\mathrm{P}<0.05$.

an increased risk of SPMs involving the esophagus, small intestine, pancreas, kidney, and thyroid, but there was a decreased risk of SPM involving the prostate (Table 4).

\section{Stage of primary $G C$}

Tumor stage has been shown to be closely associated with the long-term outcomes of patients. However, it is still unclear whether the incidence of SPMs varies according to tumor stage. Here, the SIRs for three subgroups, including localized-, regional-, and distal-stage groups, were compared. The results showed that patients in the localized and regional stages were at higher risk of developing SPMs at all sites than patients in the distal stage (Figure 1C). GC patients in the localized stage had a higher risk of developing secondary malignancies of the esophagus, stomach, small intestine, and thyroid but a lower risk of developing SPMs in the rectosigmoid junction and rectum than GC patients in the other two stages. For patients in the regional stage, the risk of SPMs of the esophagus, stomach, colon, pancreas, and thyroid was increased. Although the overall risk was not reasonably increased in patients diagnosed with disease at the distant stage, the risk of SPMs in the stomach was increased (Table 5).

\section{Duration since primary GC diagnosis}

From 6 to 11 months after primary GC diagnosis, there was a decreased risk of developing SPMs in all sites except the small intestine and thyroid (Figure 1D). After 11 months of latency, the risk was significantly increased in all sites compared with that in the general population. The highest risk was between $60-119$ months since primary GC diagnosis. At 120 months since the primary GC diagnosis, there were still dramatically increased risks for cancers of the stomach, gallbladder, and pancreas. In contrast, the risk of SPM of the prostate was relatively decreased at 120 months since primary GC diagnosis (Table 6).

\section{Discussion}

In this study, we observed that GC survivors had higher risks of developing SPM than the general US population, which is consistent with a previous study (11). In these GC survivors, increased risks for SPMs involving the stomach, small intestine, esophagus, colon, pancreas, and thyroid were observed. In contrast, the risk of SPMs of the female breast and prostate were decreased. Accumulating studies have suggested that a family history of cancer, genetic 
Table 3 SIRs of SPMs in GC patients, by age

\begin{tabular}{|c|c|c|c|}
\hline SPMs & \multicolumn{3}{|c|}{ Age, years } \\
\hline All sites & $2.69(1.82-3.84)^{\star}$ & $1.59(1.45-1.75)^{\star}$ & $1.00(0.95-1.05)$ \\
\hline Esophagus & $0.00(0.00-60.94)$ & $4.52(2.53-7.45)^{\star}$ & $2.02(1.42-2.80)^{*}$ \\
\hline Stomach & $50.19(22.95-95.28)^{*}$ & $13.11(10.18-16.63)^{*}$ & $3.28(2.76-3.87)^{\star}$ \\
\hline Colon & $10.41(3.38-24.30)^{*}$ & $1.39(0.88-2.09)$ & $1.12(0.94-1.31)$ \\
\hline Rectosigmoid junction and rectum & $5.63(0.68-20.32)$ & $1.62(0.91-2.67)$ & $0.90(0.64-1.22)$ \\
\hline Liver & $5.77(0.15-32.12)$ & $1.43(0.69-2.64)$ & $1.11(0.75-1.60)$ \\
\hline Gallbladder & $0.00(0.00-235.00)$ & $1.75(0.04-9.74)$ & $0.96(0.31-2.25)$ \\
\hline Female breast & $0.73(0.09-2.64)$ & $1.13(0.81-1.54)$ & $0.69(0.55-0.86)^{*}$ \\
\hline Cervix uteri & $2.98(0.08-16.63)$ & $0.58(0.01-3.22)$ & $1.23(0.40-2.87)$ \\
\hline Corpus uteri & $0.00(0.00-10.05)$ & $0.90(0.36-1.85)$ & $0.79(0.46-1.27)$ \\
\hline Ovary & $0.00(0.00-16.16)$ & $1.83(0.67-3.98)$ & $1.33(0.79-2.10)$ \\
\hline Prostate & $0.00(0.00-6.38)$ & $0.83(0.63-1.08)$ & $0.78(0.69-0.89)^{*}$ \\
\hline Kidney & $2.73(0.07-15.19)$ & $1.97(1.19-3.08)^{*}$ & $1.04(0.74-1.43)$ \\
\hline Urinary bladder & $5.49(0.14-30.59)$ & $1.66(0.98-2.62)$ & $1.00(0.81-1.22)$ \\
\hline Brain & $0.00(0.00-17.61)$ & $1.32(0.36-3.37)$ & $0.49(0.18-1.07)$ \\
\hline
\end{tabular}

${ }^{*}, \mathrm{P}<0.05$.

factors, and intensive treatment affect the development of SPMs in other organs. Although cooccurrence of GC and other cancers can be due to certain disorders, such as microsatellite instability, E-cadherin defects, germline mutations, and TP53 and RAS mutations, the detailed mechanism of the development of SPM is still not clear (12-14).

Age was one of the most critical risk factors for GC $(15,16)$. Here, we assessed the risk of developing SPM after GC in different age subgroups. The results showed that patients aged between 20 and 39 years had an increased risk compared with elderly patients. In terms of potential reasons for the observed increased risk in young patients, genetic susceptibility in cases with familial cancer syndromes, such as Lynch syndrome, might contribute to the higher risk of SPM in young patients. Patients with Lynch syndrome always have onset at a younger age and present with an increased risk of developing malignancies of the small intestine, colon, rectum, pancreas, and ovary $(17,18)$.

The influence of race on GC incidence and survival has been noted for several decades $(19,20)$. However, few studies have focused on the risk of developing SPMs among different races. Here, we found that American Indian and 
Table 4 SIRs of SPMs in GC patients, by race

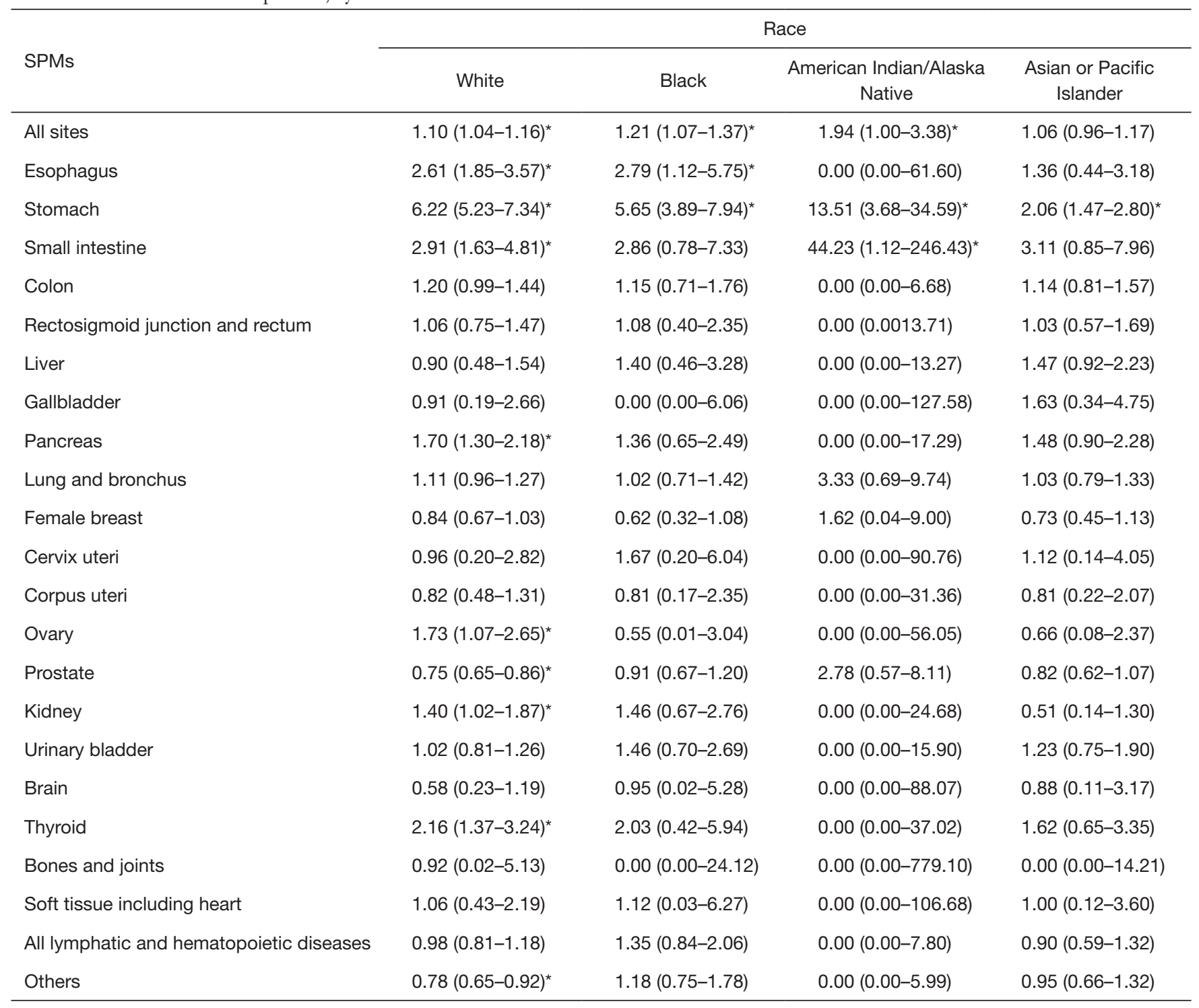

*, $\mathrm{P}<0.05$

Alaska Native GC patients tended to have the greatest risk of developing SPMs, followed by black and white patients. The risk for Asian or Pacific Islanders was not increased compared with that in the general population. This phenomenon might reflect the differences in genetic predisposition and environmental exposure, as well as in cancer screening and early treatment of precancerous lesions. Future investigations are required to explore the specific reasons for these differences.

Tumor stage is considered an important prognostic factor for cancer patients. However, it is still unclear whether the risk of developing SPM is similar in GC patients with different tumor stages. In the present study, we found that patients in the localized stage had a significantly higher risk of developing SPMs than patients in the regional and distant stages. One possible reason is that patients in the localized stage generally have longer survival durations, providing them with more chances to develop SPMs during their lifetime.

The risk of developing SPMs increased with increasing duration since primary GC diagnosis, especially in the period from 60 to 119 months. It is well known that most 
Table 5 SIRs of SPMs in GC patients, by stage

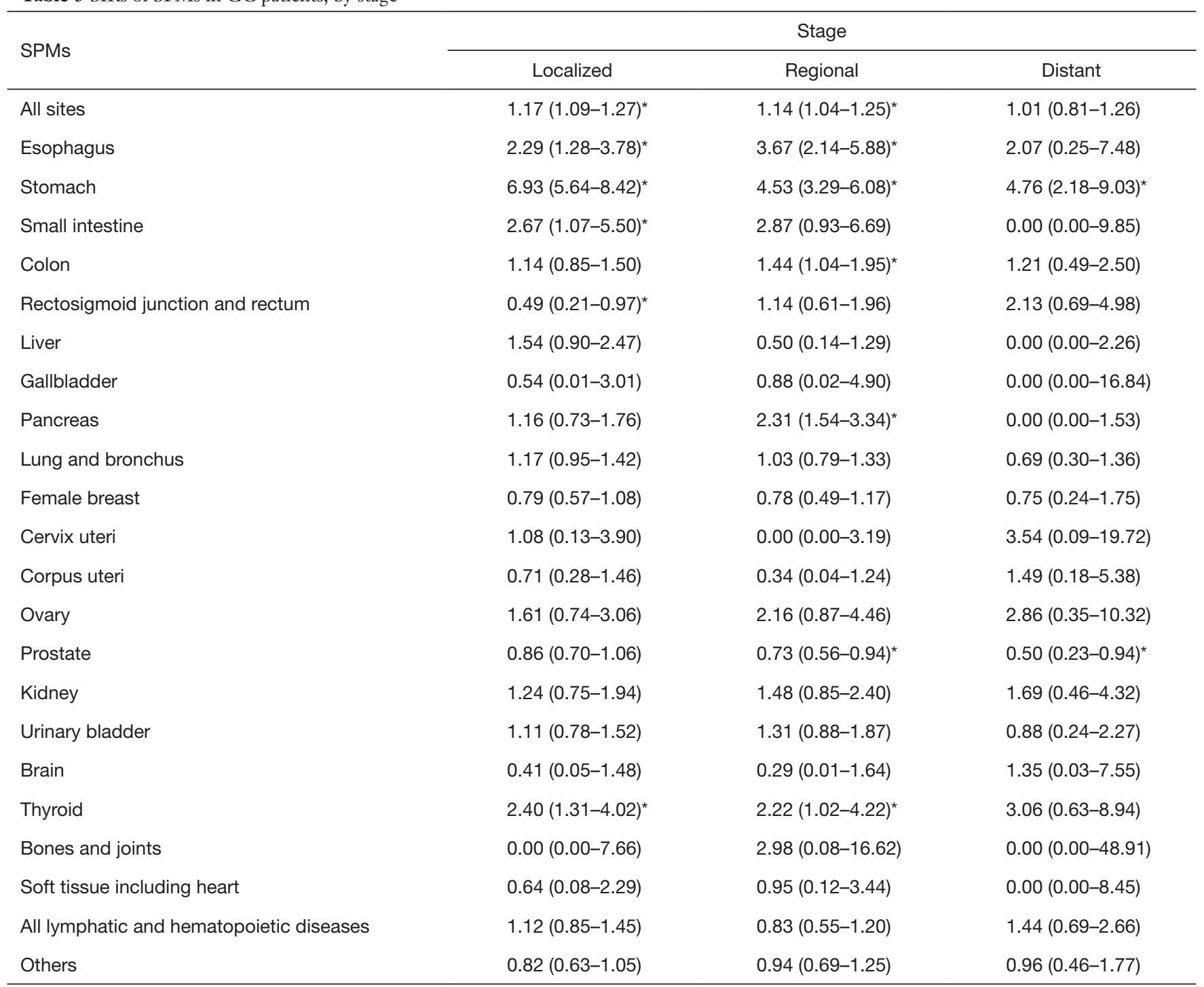

*, $\mathrm{P}<0.05$.

recurrences and metastases occur within 60 months after the diagnosis of cancer, and postoperative follow-up during the first 60 months is a crucial for cancer survivors. However, according to our results, many GC survivors present with a high risk of developing SPMs after 60 months of follow-up. Therefore, it is vital to screen for SPMs during the longterm follow-up of GC survivors. Early diagnosis of SPMs could provide GC survivors with prolonged survival and better quality of life $(21,22)$.

In the present study, there are several potential limitations. First, it is well known that the occurrence of SPMs is the result of a combination of many factors. Not only lifestyle, genetic susceptibility, and environmental factors but also the use of chemotherapy and radiotherapy for primary cancer treatment may increase the risk of developing an SPM (23). However, the SEER database lacks some patient and therapy information, including lifestyle factors, therapeutic strategies used for the primary GC, and comorbidities of GC patients. Additionally, the number of statistical tests performed to estimate the risk of SPMs by tumor type increases the likelihood of observing a false positive significant finding, suggesting that these results should be interpreted with some caution. Finally, misclassification of the treatment received may have led to 
Table 6 SIRs of SPMs in GC patients, by latency

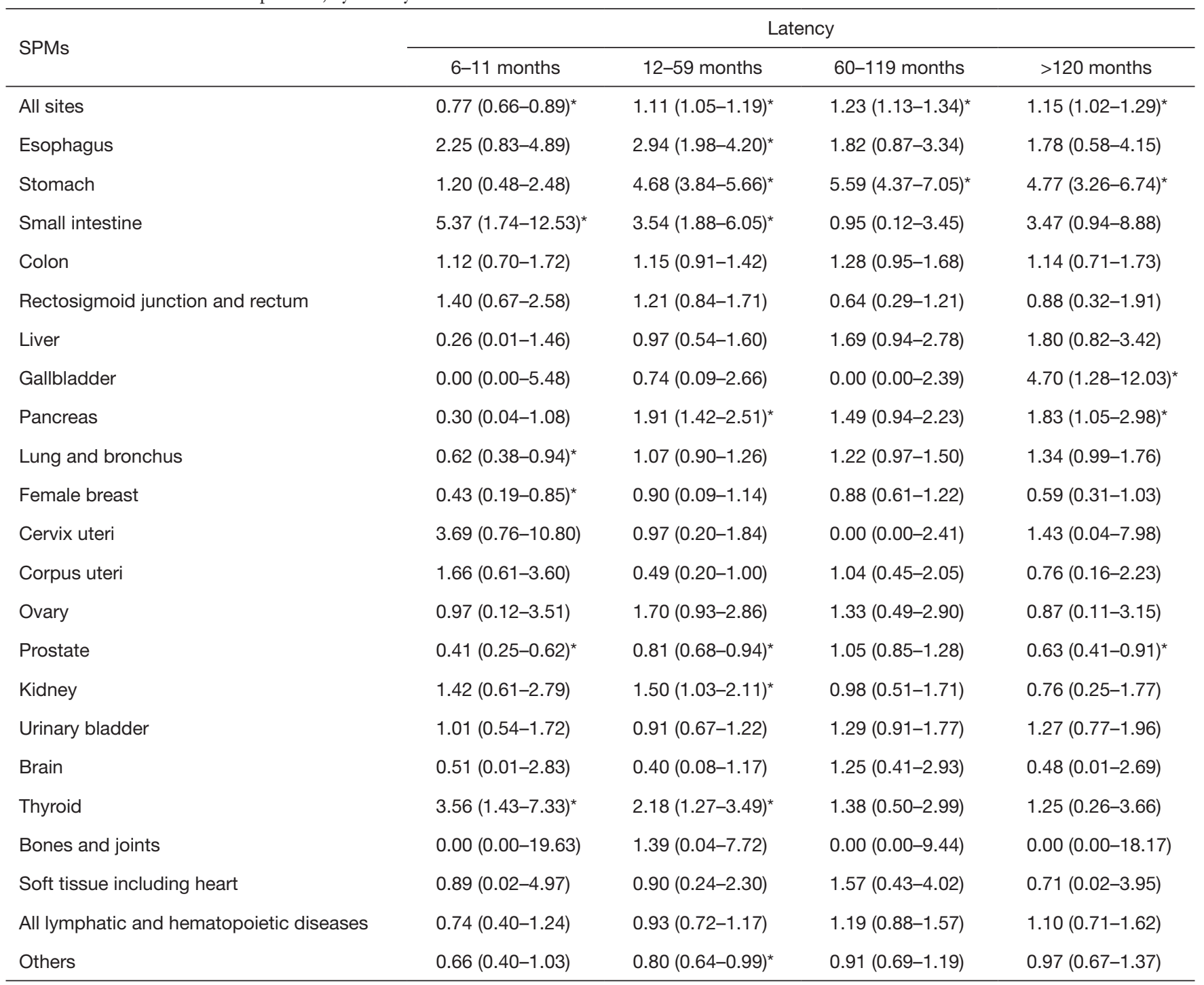

*, $\mathrm{P}<0.05$.

an underestimate of the actual risk of SPMs associated with radiation therapy (24).

\section{Conclusions}

To conclude, GC survivors are at higher risk of developing certain malignancies, particularly malignancies originating in the gastrointestinal tract. Young GC patients have a much higher risk of developing SPMs than older patients. Significant racial disparities also exist: Asian and Pacific Islander patients are less likely to experience SPMs than their counterparts of other races. Localized and regional
GC patients are more likely to develop SPMs than their distant-stage counterparts. SPMs are most likely to occur during the period from 60 to 119 months after the initial GC diagnosis. All of these findings have significant clinical implications for the effective prevention and continuous surveillance of SPMs among GC survivors.

\section{Acknowledgments}

The authors acknowledge the efforts of the Surveillance, Epidemiology, and End Results (SEER) Program tumor registries in the creation of the SEER database. The 
interpretation of these data is the sole responsibility of the authors.

Funding: None.

\section{Footnote}

Reporting Checklist: The authors present the study in accordance with the STROBE reporting checklist. Available at http://dx.doi.org/10.21037/tcr-20-1105

Conflicts of Interest: All authors have completed the ICMJE uniform disclosure form (available at http://dx.doi. org/10.21037/tcr-20-1105). The authors have no conflicts of interest to declare.

Ethical Statement: The authors are accountable for all aspects of the work in ensuring that questions related to the accuracy or integrity of any part of the work are appropriately investigated and resolved. The study was conducted in accordance with the Declaration of Helsinki (as revised in 2013).

Open Access Statement: This is an Open Access article distributed in accordance with the Creative Commons Attribution-NonCommercial-NoDerivs 4.0 International License (CC BY-NC-ND 4.0), which permits the noncommercial replication and distribution of the article with the strict proviso that no changes or edits are made and the original work is properly cited (including links to both the formal publication through the relevant DOI and the license). See: https://creativecommons.org/licenses/by-nc-nd/4.0/.

\section{References}

1. Torre LA, Bray F, Siegel RL, et al. Global cancer statistics, 2012. CA Cancer J Clin 2015;65:87-108.

2. Malvezzi M, Bonifazi M, Bertuccio P, et al. An age-periodcohort analysis of gastric cancer mortality from 1950 to 2007 in Europe. Ann Epidemiol 2010;20:898-905.

3. Bertuccio P, Chatenoud L, Levi F, et al. Recent patterns in gastric cancer: a global overview. Int J Cancer 2009;125:666-73.

4. Sisti G, Kanninen TT, Asciutti S, et al. Rate of second primary tumors following diagnosed choriocarcinoma: a SEER analysis (1973-2010). Gynecol Oncol 2014;134:90-5.

5. MacCarthy A, Bayne AM, Brownbill PA, et al. Second and subsequent tumours among 1927 retinoblastoma patients diagnosed in Britain 1951-2004. Br J Cancer 2013;108:2455-63.

6. Guan X, Jin Y, Chen Y, et al. The Incidence Characteristics of Second Primary Malignancy after Diagnosis of Primary Colon and Rectal Cancer: A Population Based Study. PLoS One 2015;10:e143067.

7. Kim C, Bi X, Pan D, et al. The risk of second cancers after diagnosis of primary thyroid cancer is elevated in thyroid microcarcinomas. Thyroid 2013;23:575-82.

8. Coyte A, Morrison DS, McLoone P. Second primary cancer risk - the impact of applying different definitions of multiple primaries: results from a retrospective populationbased cancer registry study. BMC Cancer 2014;14:272.

9. Irimie A, Achimas-Cadariu P, Burz C, et al. Multiple primary malignancies--epidemiological analysis at a single tertiary institution. J Gastrointestin Liver Dis 2010;19:69-73.

10. Hankey BF, Ries LA, Edwards BK. The surveillance, epidemiology, and end results program: a national resource. Cancer Epidemiol Biomarkers Prev 1999;8:1117-21.

11. Shah BK, Khanal A, Hewett Y. Second Primary Malignancies in Adults with Gastric Cancer - A US Population-Based Study. Front Oncol 2016;6:82.

12. Ohtani H, Yashiro M, Onoda N, et al. Synchronous multiple primary gastrointestinal cancer exhibits frequent microsatellite instability. Int J Cancer 2000;86:678-83.

13. Pharoah PD, Guilford P, Caldas C, et al. Incidence of gastric cancer and breast cancer in $\mathrm{CDH} 1$ (E-cadherin) mutation carriers from hereditary diffuse gastric cancer families. Gastroenterology 2001;121:1348-53.

14. Yang HK, Linnoila RI, Conrad NK, et al. TP53 and RAS mutations in metachronous tumors from patients with cancer of the upper aerodigestive tract. Int J Cancer 1995;64:229-33.

15. Tavares A, Gandra A, Viveiros F, et al. Analysis of clinicopathologic characteristics and prognosis of gastric cancer in young and older patients. Pathol Oncol Res 2013;19:111-7.

16. Imai K, Mitsunaga A, Araida T, et al. Advanced gastric cancer associated with nodular gastritis in a young patient. Clin J Gastroenterol 2009;2:275-8.

17. Lucci S, Pasta V, Arcieri S, et al. Hereditary nonpolyposis colorectal cancer (Lynch syndrome): a review of the literature and case reports. G Chir 2000;21:177-87.

18. Vasen HF, Wijnen JT, Menko FH, et al. Cancer risk in families with hereditary nonpolyposis colorectal cancer diagnosed by mutation analysis. Gastroenterology 
1996;110:1020-7.

19. Howard JH, Hiles JM, Leung AM, et al. Race influences stage-specific survival in gastric cancer. Am Surg 2015;81:259-67.

20. Taylor VM, Ko LK, Hwang JH, et al. Gastric cancer in Asian American populations: a neglected health disparity. Asian Pac J Cancer Prev 2014;15:10565-71.

21. Buyukasik O, Hasdemir AO, Gulnerman Y, et al. Second primary cancers in patients with gastric cancer. Radiol Oncol 2010;44:239-43.

Cite this article as: Li S, Luo Y, Hou Q, Chu H, Zheng H. Incidence features of second primary malignancy among gastric cancer survivors, 1992-2012. Transl Cancer Res 2020;9(11):70017011. doi: $10.21037 /$ tcr-20-1105
22. Kim JW, Jang JY, Chang YW, et al. Clinical features of second primary cancers arising in early gastric cancer patients after endoscopic resection. World J Gastroenterol 2015;21:8358-65.

23. Birgisson H, Påhlman L, Gunnarsson U, et al. Occurrence of second cancers in patients treated with radiotherapy for rectal cancer. J Clin Oncol 2005;23:6126-31.

24. Buiatti E, Crocetti E, Acciai S, et al. Incidence of second primary cancers in three Italian population-based cancer registries. Eur J Cancer 1997;33:1829-34. 